\title{
The effect of anti-VEGF drugs (bevacizumab and aflibercept) on the survival of patients with metastatic colorectal cancer (mCRC)
}

REVIEW

This article was published in the following Dove Press journal:

OncoTargets and Therapy

12 April 2012

Number of times this article has been viewed

\section{Kuifeng $\mathrm{He}^{1}$ \\ Binbin $\mathrm{Cui}^{2}$ \\ Guangliang $\mathrm{Li}^{\prime}$ \\ Haohao Wang' \\ Ketao Jin' \\ Lisong Teng'}

'Department of Surgical Oncology, First Affiliated Hospital, ${ }^{2}$ Department of Surgical Oncology, Sir Run Run Shaw Hospital, College of Medicine, Zhejiang University, Hangzhou,

Zhejiang, China
Correspondence: Lisong Teng Department of Surgical Oncology, First Affiliated Hospital, College of Medicine, Zhejiang University, 79 Qingchun Road, Hangzhou, Zhejiang 310003 , China

Tel +86 57| $87068873 /+86$ I36 66676918

Fax +86-57I-87236628

Email Isteng@zju.edu.cn

\begin{abstract}
Significant progression has been achieved in the treatment of metastatic colorectal cancer (mCRC) in recent years. This has been partly attributed to successfully incorporating new drugs into combination chemotherapy. In addition to the traditional cytotoxic chemotherapeutic agents, molecularly targeted agents began to play an important role in the treatment of advanced solid tumors. To date, two classes of molecularly targeted agents have been approved for treatment of patients with mCRC: (1) antivascular endothelial growth factor (anti-VEGF) agents (such as bevacizumab and aflibercept) and (2) antiendothelial cell growth factor receptor (anti-EGFR) agents (such as cetuximab and panitumumab). Aflibercept is a new member of anti-VEGF agents which has demonstrated efficacy for treatment of mCRC. With the commencement of clinical trials and basic research into aflibercept, more data from the bedside and the bench have been obtained. This review will outline the application of anti-VEGF agents by reviewing clinic experiences of bevacizumab and aflibercept, and try to add perspectives on the use of anti-VEGF agents in mCRC.
\end{abstract}

Keywords: chemotherapy, tumors, antiangiogenic

\section{Introduction}

Colorectal cancer (CRC) is a critical health problem. With more than 800,000 new cases diagnosed every year, CRC is the third most-common cancer worldwide. ${ }^{1}$ Although mortality from CRC has decreased slightly over the past decade as a result of earlier diagnosis, approximately $20 \%$ of patients advance to metastatic disease. ${ }^{2}$ When it comes to the stage where it cannot be surgically removed, the prognosis of this disease is poor.

Rapid advances in tumor biology are promising a new era of molecularly targeted cancer therapy. Bevacizumab is a monoclonal antibody targeted at vascular endothelial growth factor (VEGF). By preventing VEGF binding to their receptors (VEGFRs), it can inhibit tumor angiogenesis. A phase III trial revealed that the overall survival was extended from 15.6 months to 20.3 months $(P<0.001)$ when bevacizumab was added to irinotecan plus fluorouracil/leucovorin (IFL) for treatment of metastatic colorectal cancer patients (mCRC). ${ }^{3}$ The results have been promising and have assisted in the mechanisms of tumor angiogenesis being further understood, ${ }^{4,5}$ with more than 50 new drugs with anti-angiogenic activity having been developed. ${ }^{6}$

Recently, aflibercept (VEGF-Trap), a fusion protein with high VEGF affinity, has extended progression-free survival and overall survival of $\mathrm{mCRC}$ patients in a phase III trial (VELOUR), ${ }^{7}$ which included aflibercept with irinotecan/5-FU as second-line chemotherapy. 


\section{Anti-VEGF rationale for $\mathrm{MCRC}$}

Neovascularization is a critical process in solid tumor progression. Without vascular provided oxygen and nutrients, tumors struggle to grow beyond $2 \mathrm{~mm}$ in diameter. ${ }^{89}$

Blood vessel formation in tumors involves several different processes: the classic endothelial sprouting process, vessel co-option, intussusceptive microvascular growth (IMG), glomeruloid angiogenesis, endothelial progenitor cell mobilization, and vasculogenic mimicry. ${ }^{5}$ In most conditions, new vascular blood flows were formed by endothelial-sprouting from existing vessels, called angiogenesis. Neovascularization is regulated by the balance of pro- and anti-angiogenic factors. ${ }^{4}, 10$ VEGF family members are believed to be the most important proangiogenic factors. VEGF-A is thought to be the key controller of the angiogenic switch. ${ }^{11,12}$ VEGF promotes angiogenesis by stimulating endothelial cell proliferation and migration, altering blood vessel permeability, and controlling the functional and morphological form of these vessels.

Further, VEGF can play a role in the non-sprouting vascularization processes previously mentioned. ${ }^{5,13}$ For example, it can recruit marrow-derived circulating endothelial cell progenitors (CEPs) to create vascular formations. In tumors, VEGF-induced vessels are structurally immature and functionally abnormal, which is characterized by irregular dilated lumina, tortuous shape, pericyte deficiency, and hyper permeability. ${ }^{10}$ This abnormal vasculature leads to increased interstitial fluid pressure (IFP), as well as deficiency of nutrients and oxygen delivery, which triggers further VEGF production. ${ }^{14}$ High IFP can further hinder the delivery of nutrients and oxygen, as well as cytotoxic drugs. ${ }^{15}$ Studies have revealed that VEGF expression is elevated in a wide variety of tumor types including CRC. ${ }^{16,17}$ Hyper expression of VEGF has also been demonstrated to be associated with the progression, invasion, and metastasis of CRC. ${ }^{16,18}$

VEGF is considered a key target for treatment of solid tumors and this idea has been proven by bevacizumab, which is a humanized monoclonal antibody against VEGF-A. Validated by testing in various animal models, antiangiogenic drugs (including anti-VEGF agents) work via several mechanisms, such as increasing the delivery of cytotoxic drugs via vessel normalization. ${ }^{19}$ An additional hypothesis is that antiangiogenic drugs can control tumor cell repopulation during the chemotherapy drug-free break period. A third hypothesis is that inhibiting the mobilization of marrow derived circulating endothelial cells (CECs) or their progenitors (CEPs) is an important mechanism for antiangiogenic drugs to slow tumor growth and sensitize chemotherapy. ${ }^{20,21}$

\section{Clinical evidence of anti-VEGF strategy in $\mathrm{MCRC}$ treatment}

Bevacizumab is the most clinically advanced anti-VEGF agent and the first one to receive approval for first- and second-line treatment of mCRC. The experience of bevacizumab is indicative of the value of anti-VEGF strategies in the treatment of CRC. Clinical experience of anti-VEGF treatment of mCRC provided by bevacizumab was reported as follows:

1. As a single agent, it only provided modest response rates but demonstrated significant efficacy when combined with other strategies. ${ }^{22}$

2. It demonstrated efficacy in combination with all the basic chemotherapeutic regimens, but failed to provide benefits in combination with anti-EGFR agents.

3. More and more evidence suggests that continuous administration can provide survival benefits, even after disease progression.

4. Application in postoperative adjuvant therapy is not advisable as there is no efficacy at increasing overall survival.

After the pivotal trial proved that it can prolong overall survival, bevacizumab has been extensively incorporated into various chemotherapy protocols to manage mCRC. The most commonly used first-line chemotherapy regimens for CRC in clinical practice (FOLFOX [LV, 5-FU, oxaliplatin], FOLFIRI [LV, 5-FU, irinotecan], and XELOX [capecitabine, oxaliplatin]), ${ }^{23}$ have all been proven to benefit from the combination with bevacizumab (Table 1).

\section{Combination with irinotecan contained regimen}

Combined bevacizumab with irinotecan contained regimen IFL was verified firstly in the phase III trial which led to the approval of bevacizumab in the treatment of mCRC. The bolus IFL regimen, which was proven later in the BICC-C trial to be inferior, had increased toxicity and less efficacy ${ }^{24}$ and was replaced by the infusion regimen FOLFIRI. It is this trial also that proved the superior clinical activity of FOLFIRI over a modified IFL (mIFL) regimen with prolonged median progression-free survival (PFS) (7.6 versus 5.9 months). Moreover, median overall survival (OS) was significantly prolonged for the FOLFIRI plus bevacizumab group, compared with the mIFL plus bevacizumab group (median OS 28.0 versus 19.2 months; $P=0.037) .{ }^{25}$ 
Table I Critical trials of bevacizumab for metastatic colorectal cancer

\begin{tabular}{|c|c|c|c|c|}
\hline Basic chemotherapy & Clinic trial (references) & Bevacizumab schedule & Median PFS & Median OS \\
\hline \multicolumn{5}{|l|}{ Irinotecan } \\
\hline IFL & AVF2 $107^{3}$ & $5 \mathrm{mg} / \mathrm{kg} \mathrm{IV}$, every 2 weeks & $\begin{array}{l}\text { I0.6 vs } 6.2 \text { months, } \\
P<0.00 \text { I }\end{array}$ & 20.3 vs 15.6 months, $P<0.00$ I \\
\hline FOLFIRI or mIFL & $\mathrm{BICC}-\mathrm{C}^{24,25, \mathrm{a}}$ & $\begin{array}{l}\text { FOLFIRI+Bev: } 5 \text { mg/kg IV, } \\
\text { every } 2 \text { weeks; } \\
\text { mIFL+Bev: } 7.5 \text { mg/kg IV, } \\
\text { every } 3 \text { weeks }\end{array}$ & 7.6 vs 5.9 months & $\begin{array}{l}23.1 \text { vs } 17.6 \text { months } \\
\text { (without Bev); } 28.0 \text { vs } \\
\text { I9.2 months (with Bev) }\end{array}$ \\
\hline \multicolumn{5}{|l|}{ Oxaliplatin } \\
\hline FOLFOX or XELOX & NOI6966 $26, b$ & $7.5 \mathrm{mg} / \mathrm{kg} \mathrm{IV}$, every 3 weeks & $\begin{array}{l}9.4 \text { vs } 8.0 \text { months, } \\
P=0.0023\end{array}$ & 21.3 vs 19.9 months; $P=0.077$ \\
\hline FOLFOX4 & $\mathrm{E} 3200^{27}$ & $10 \mathrm{mg} / \mathrm{kg} \mathrm{IV}$, every 14 days & $\begin{array}{l}7.2 \text { vs } 4.8 \text { months; } \\
P<0.000 \text { I }\end{array}$ & I 2.5 vs 10.7 months; $P=0.0024$ \\
\hline FOLFOX or bFOL or & TREE-I and & FOLFOX and bFOL: & $8.7,6.9$, and 5.9 vs & 19.2, 17.9, and 17.2 vs 26.1 , \\
\hline CAPOX & TREE-2 (with Bev) ${ }^{15, c}$ & $\begin{array}{l}5 \mathrm{mg} / \mathrm{kg} \text { IV, every } 2 \text { weeks; } \\
\text { CapeOx: } 7.5 \mathrm{mg} / \mathrm{kg} \mathrm{IV} \text {, } \\
\text { every } 3 \text { weeks }\end{array}$ & $9.9,8.3$, and 10.3 months & 20.4 , and 24.6 months \\
\hline \multicolumn{5}{|l|}{ 5-FU } \\
\hline 5-FU/LV & $\begin{array}{l}\text { AVF0780, AVF2107 } \\
\text { and AVF219228,29,d }\end{array}$ & $5 \mathrm{mg} / \mathrm{kg} \mathrm{IV}$, every 2 weeks & $\begin{array}{l}8.8 \text { vs } 5.6 \text { months, } \\
P \leq 0.00 I\end{array}$ & 17.9 vs 14.6 months, $P=0.008$ \\
\hline
\end{tabular}

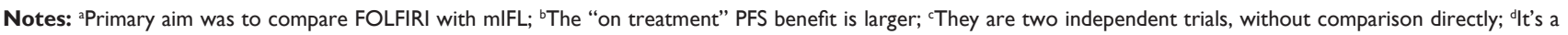
combined analysis of the three trials.

Abbreviations: IV, intravenous; Bev, bevacizumab; mIFL, modified IFL.

\section{Combination with oxaliplatin-based regimen}

When bevacizumab was incorporated into the oxaliplatin-based regimen, bevacizumab failed to increase response rates (38\% in both arms) or survival (19.9 months versus 21.3 months, $P=0.077$, compared with either combination alone) of metastasis mCRC, with either FOLFOX or XELOX in N016966 trial. This may be caused by frequent disruption, as "on treatment" PFS benefit is significant. ${ }^{26}$ In the following Eastern Cooperative Oncology Group trial (ECOG 3200), the use of bevacizumab with FOLFOX in second-line treatment of metastatic colon cancer resulted in significantly improved PFS (7.3 months versus 4.7 months) and median survival (12.9 months versus 10.8 months) compared with FOLFOX alone. ${ }^{27}$

\section{Combination with 5-FU-based regimen}

As there are some patients that can't receive multi-drug chemotherapy due to tolerance problems or other reasons, they may be treated with single 5-Fu. A combined analysis of the results from three different studies showed that the combination with bevacizumab is superior to $5-\mathrm{FU}$ alone..$^{28,29}$

\section{Continued application in refractory disease}

The nonrandomized prospective observational study, BRiTE (Bevacizumab Regimens: investigation of Treatment Effects and safety), firstly evaluated the efficacy of bevacizumab in the post-progressive treatment for mCRC. Results showed that it provided significant efficacy and appropriate safety. The OS from the time of progression for the group that received postprogressive therapy with the bevacizumab containing regimen was 19.2 months, while it was 9.5 months for the group that received post-progressive therapy without bevacizumab. ${ }^{30,31}$ Samelis et $\mathrm{l}^{32}$ recently reported on 21 patients included in their retrospective study results, in which bevacizumab was continuously applied on these mCRC patients, who had received at least one course of irinotecan-based or oxaliplatinbased chemotherapy with bevacizumab, after disease progression: the median OS was $23+$ months, and median time to progression (TTP) was 17 months.

\section{Combination with EGFR inhibitor}

Two classes of targeted agent have been validated in the treatment of $\mathrm{mCRC}$ in combination with chemotherapy: anti-VEGF agent and EGFR inhibitor. Of note, there were studies that indicated that EGFR had a potent effect on tumorassociated angiogenesis and combined treatment with EGFR and VEGF signaling inhibitors had at least additive antitumor activity. ${ }^{15,33,34}$ The use of dual biologic therapies in combination with cytotoxic chemotherapy has been evaluated by two phase II studies, the PACCE and CAIRO 2. However, the results are only of impaired benefits and increased toxicity. ${ }^{35,36}$ 
The results are further supported by the recently reported Phase III trial, which compared the treatment of mFOLFOX6-B (modified FOLFOX6 + bevacizumab) with that of FOLF-CB (5-FU, leucovorin, + bevacizumab and cetuximab) for CRC patients. The results revealed that the efficacy of the dual biologic FOLF-CB group was not superior to the mFOLFOX6-B group. Patient satisfaction favored the control (mFOLFOX6-B). ${ }^{37}$ Twelve-month PFS was $45 \%$ versus $32 \%$, and median OS was 21 months versus 19.5 months.

\section{Application in postoperative adjuvant therapy}

Experimental evidence suggests that VEGF induced vasculogenesis is most important in the earlier stage of tumorigenesis and progression. Consequently, anti-VEGF agents provide their greatest benefit early in disease when tumors are small and there is minimal residual disease. ${ }^{15}$ Thus, it is a good choice to use bevacizumab in the adjuvant setting in principle. However, there is little further evidence that encourages us going forward because of the disappointing results of two phase III trials in postoperative chemotherapy (NSABP C-08 and AVANT). ${ }^{38,39}$

\section{Characteristics and application information of aflibercept}

Aflibercept is a fully human recombinant protein which is constructed by fusing the second extracellular domain of VEGFR1 and the third extracellular domain of VEGFR2 to the Fc segment of immunoglobulin $\mathrm{G} 1 .{ }^{40}$ Other than bevacizumab and ranibizumab binding all isomers of the VEGF-A, aflibercept also binds VEGF-B and placental growth factor. ${ }^{41,42}$ Early studies in murine models revealed that aflibercept can bind with very high affinity $(\approx 1 \mathrm{pM})$ to multiple isoforms of VEGF, which was superior to other anti-VEGF reagents. ${ }^{43}$ Being studied on various animal model, aflibercept has been proven to act on endothelial cells, pericytes, and even the vascular basement membrane or VEGF receptor, resulting in the inhibition of new vessel growth and remodeling of preexisting ones, which had been detected in bevacizumab. ${ }^{43,44}$ Remodel effects usually present as the normalization of tumor vasculature, which is believed to be the mechanism of anti-VEGF agents enhancing chemotherapy efficacy. ${ }^{45}$

As we detailed earlier, ${ }^{44}$ in mouse model studies, aflibercept can decrease vascular growth and density, and suppress tumor growth, when combined with HER2 antibodies (trastuzumab) to treat breast carcinoma; enhance the efficacy of radiation for treating neuroblastoma xenografts; reduce tumor burden; and inhibit metastasis of ovarian cancer in combination with paclitaxel. ${ }^{46}$ Completed phase I trials suggest that aflibercept is well tolerated and the advised dose is $4 \mathrm{mg} / \mathrm{kg}{ }^{44,47}$ The most common drug toxicities reported in phase I/II trials include fatigue, proteinuria, hypertension, nausea, and lymphopenia. ${ }^{48-50}$ Grade 3 to 4 toxicity incidence is extremely rare. It's notable that thromboembolic events are less observed, which are frequent in treatment with bevacizumab. If this difference is real, it may be attributed to the $1: 1$ bind ratio with VEGF and full human protein sequences. ${ }^{51}$

Since anti-VEGF monotherapy showed poor results in previous trials, anti-VEGF treatment is often combined with other strategies. Most of the phase II and III trials evaluated aflibercept efficacy in combination with chemotherapy. To date, a range of tumor types have been treated with aflibercept in clinical trials, including ovarian cancer, non-small cell lung adenocarcinoma, prostate carcinoma, and mCRC. ${ }^{41,52}$

One Phase III trial (VELOUR) is evaluating aflibercept as a second line treatment for $\mathrm{mCRC}$ in combination with FOLFIRI. In total, 1226 patients with mCRC received FOLFIRI and either aflibercept $(4 \mathrm{mg} / \mathrm{kg}$ ) or a placebo every 2 weeks after failure of one oxaliplatin-based therapy. PFS was 6.90 months versus 4.67 months (hazard ratio [HR] $0.758, P=0.00007)$ and objective response rate (ORR) $19.8 \%$ versus $11.1 \%(P=0.0001)$. Median OS was 13.50 months for aflibercept arm and 12.06 months for placebo arm (HR 0.817, $P=0.0032$ ). Treatment discontinuation for adverse events occurred in $26.6 \%$ and $12.1 \%$ in aflibercept arm and placebo arm, respectively. ${ }^{7}$

Recently, at the Deutsche Bank BioFEST conference, the preliminary results of the Phase II trial (AFFIRM), in which aflibercept was used with modified FOLFOX6 as first line treatment for patients with $\mathrm{mCRC}$, has been disclosed. It showed that the PFS rate at 1 year, for patients who received aflibercept in combination with mFOLFOX6, was similar to that seen in the standard therapy arm for patients who received mFOLFOX6 alone. The toxicity profile of aflibercept was similar to what has been seen in prior trials with aflibercept and consistent with other anti-VEGF agents. The full data set should be available at future medical conferences. ${ }^{53}$ With these proofs, Regeneron's collaborator, Sanofi (Paris, France), has submitted a Biologics License Application to the US Food and Drug Administration for marketing approval of ZALTRAPTM (aflibercept) for second line treatment of $\mathrm{mCRC}$.

There is an ongoing regional phase I trial, in which aflibercept is intravenously administered in combination with FOLFIRI for Japanese patients with metastatic colorectal cancer. The primary objective is to determine the 
dose of aflibercept to be further studied in combination with FOLFIRI in Japanese patients..$^{54}$

\section{Questions and further investigations}

It's great news that a new tool is being added to the arsenal for us to fight against mCRC. However, aflibercept may encounter a similar problem to bevacizumab in clinical application. Even though earlier promising data suggested that aflibercept has a much higher VEGF binding affinity than bevacizumab, more clinical data is needed to prove that it can be more effective.

The first issue is that VEGF induced vasculogenesis is mostly important in the earlier stage in tumorigenesis and progression. ${ }^{15}$ Based on this rationale, anti-VEGF agents are expected to provide better disease control in the adjuvant setting. Paradoxically, clinical data concluded that incorporating the anti-VEGF agent bevacizumab into adjuvant regimens does not prolong disease-free survival (DFS) or OS of patients with CRC..$^{38,39}$ Thus, to make aflibercept and other anti-VEGF agents exert greater action, the effect of VEGF and the anti-VEGF results in tumor vasculogenesis or development need to be further investigated.

Based on the theory of vascular normalization, anti-VEGF agents should be used to provide a "normalization window" for chemotherapeutic drugs. That means anti-VEGF agents should be administered intermittently, to avoid hindering the delivery of chemotherapeutic drugs. However, there is evidence showing benefits of continuous or prolonged use, even after disease progression. ${ }^{55,56}$ This is not consistent with the hypothesis that continuous treatment with bevacizumab may stimulate some alternative proangiogenic signals that induce resistance to it. ${ }^{14,57}$ Aflibercept studies should also answer the question of whether anti-VEGF agents should be continuously given in the full schedule of mCRC treatment.

Both anti-VEGF agents and EGFR inhibitors have demonstrated efficacy for the treatment of $\mathrm{mCRC}$ and crossover effects were observed between EGFR and VEGF signals. . $^{33,34}$ However, the dual biologic therapy in combination with chemotherapeutic regimens failed to demonstrate effectiveness in the PACCE and CAIRO 2 study. ${ }^{35,36}$ indicating that dual aflibercept and anti-EGFR therapy should not be used in combination with cytotoxic chemotherapy as a routine treatment in the front-line setting. We are expecting the results of an alternative combination in a phase III study, which combined chemotherapy and bevacizumab with or without erlotinib to treat patients with metastatic colorectal cancer (that cannot be removed by surgery). ${ }^{58}$ Alternative combinations can also be considered for other protocols or drugs, for example, metronomic chemotherapy (MCT) or vascular disrupting agents (VDA), both of which target blood vessels in tumors, with the effect on CEPs. ${ }^{59,60} \mathrm{~A}$ combination of the two with anti-VEGF agents may be suitable for mCRC. What's more, sequential or alternate use of them and anti-VEGF drugs may avoid resistance induced by a single agent.

It is clear the benefit varied when bevacizumab was tested in different trials. This may have been caused by drugs or regimens that are more appropriately combined with anti-VEGF agents or caused by different patient's genetic backgrounds. Unfortunately, to date, there is no validated biomarker that can be used to select patients. ${ }^{61}$ Plasma angiogenic molecules, such as VEGF, placenta growth factor (PIGF), soluble VEGFR-2, and basic fibroblast growth factor (bFGF) were most often studied for their significant level-change during the bevacizumab application, ${ }^{62}$ but hitherto, none of them have been proven to be of predictive value. In recent years, viable CECs and CEPs (which play an important role in tumor angiogenesis) have frequently been studied. Prior studies have revealed anti-angiogenic agents have potential inhibitory effects on mobilization or proliferation of endothelial progenitor cells (EPCs) in tumor tissue and CEC and CEP numbers have the potential to become surrogate biomarkers for predicting and monitoring drug activity. ${ }^{63}$ Therefore, clarifying the mechanism of EPCs in angiogenesis will facilitate the application of bevacizumab, aflibercept, and other anti-VEGF agents. Conversely, clinically confirming whether there are some drugs or regimens that are more suitable for combination with anti-VEGF agents is another urgent task.

\section{Conclusion}

As anti-VEGF agents, aflibercept and bevacizumab are promising drugs with the potential to prolong the life of patients with $\mathrm{mCRC}$ but there are quite a few problems that need to be resolved before they significantly change the outcome of mCRC. To this end, there is still a long way to go in antiangiogenesis therapy-related basic, clinical, and translational studies.

\section{Acknowledgments}

This work was supported by the State Key Basic Research and Development Program of China (973 Program, Grant No 2009CB521704), National High-tech Research and Development Program of China (863 Program, Grant No 2006AA02A245), National Natural Science Foundation of China (Grant No 81000894), Zhejiang Provincial Science and Technology Projects (Grant No 2009C13021, 2011C23087), Science Research Fund of Shaoxing 
(Grant No 2011D10013) and the Science Research Fund of Zhuji (Grant No 2011CC7874).

\section{Disclosure}

The authors report no conflicts of interest in this work.

\section{References}

1. Ortega J, Vigil CE, Chodkiewicz C. Current progress in targeted therapy for colorectal cancer. Cancer Control. 2010;17(1):7-15.

2. Jemal A, Siegel R, Ward E, et al. Cancer statistics, 2008. CA Cancer J Clin. 2008;58(2):71-96.

3. Hurwitz H, Fehrenbacher L, Novotny W, et al. Bevacizumab plus irinotecan, fluorouracil, and leucovorin for metastatic colorectal cancer. N Engl J Med. 2004;350(23):2335-2342.

4. Karamysheva AF. Mechanisms of angiogenesis. Biochemistry (Mosc). 2008;73(7):751-762.

5. Döme B, Hendrix MJ, Paku S, Tóvári J, Tímár J. Alternative vascularization mechanisms in cancer: Pathology and therapeutic implications. Am J Pathol. 2007;170(1):1-15.

6. Folkman J. Is angiogenesis an organizing principle in biology and medicine? J Pediatr Surg. 2007;42(1):1-11.

7. Van Cutsem E, Tabernero J, Lakomy R, et al. ZALTRAPTM (aflibercept) Significantly Improved Survival in Previously Treated Metastatic Colorectal Cancer Patients. Annals of Oncology. 2011;22(Suppl 5):v18.

8. Folkman J. Clinical Applications of Research on Angiogenesis. $N$ Engl J Med. 1995;333(26):1757-1763.

9. Zetter BR. Angiogenesis and tumor metastasis. Annu Rev Med. 1998;49:407-424.

10. Bergers G, Benjamin LE. Tumorigenesis and the angiogenic switch. Nat Rev Cancer. 2003;3(6):401-410.

11. Hicklin DJ, Ellis LM. Role of the vascular endothelial growth factor pathway in tumor growth and angiogenesis. J Clin Oncol. 2005;23(5):1011-1027.

12. Ferrara N. Vascular endothelial growth factor as a target for anticancer therapy. Oncologist. 2004;9 Suppl 1:2-10.

13. Hicklin DJ, Ellis LM. Role of the vascular endothelial growth factor pathway in tumor growth and angiogenesis. J Clin Oncol. 2005;23(5):1011-1027.

14. Ferrara N, Hillan KJ, Gerber HP, Novotny W. Discovery and development of bevacizumab, an anti-VEGF antibody for treating cancer. Nat Rev Drug Discov. 2004;3(5):391-400.

15. Carrato A, Gallego-Plazas J, Guillen-Ponce C. Anti-VEGF therapy: a new approach to colorectal cancer therapy. Expert Rev Anticancer Ther. 2006;6(10):1385-1396.

16. Ishigami SI, Arii S, Furutani M, et al. Predictive value of vascular endothelial growth factor (VEGF) in metastasis and prognosis of human colorectal cancer. Br J Cancer. 1998;78(10):1379-1384.

17. Wong MP, Cheung N, Yuen ST, Leung SY, Chung LP. Vascular endothelial growth factor is up-regulated in the early pre-malignant stage of colorectal tumour progression. Int J Cancer. 1999;81(6): $845-850$.

18. Martins SF, Reis RM, Rodrigues AM, Baltazar F, Filho AL. Role of endoglin and VEGF family expression in colorectal cancer prognosis and anti-angiogenic therapies. World J Clin Oncol. 2011;2(6): $272-280$.

19. Jain RK. Normalization of tumor vasculature: an emerging concept in antiangiogenic therapy. Science. 2005;307(5706):58-62.

20. Kerbel RS. Antiangiogenic therapy: a universal chemosensitization strategy for cancer? Science. 2006;312(5777):1171-1175.

21. Shaked Y, Henke E, Roodhart JM, et al. Rapid chemotherapyinduced acute endothelial progenitor cell mobilization: implications for antiangiogenic drugs as chemosensitizing agents. Cancer Cell. 2008;14(3):263-273.
22. Gordon MS, Margolin K, Talpaz M, et al. Phase I safety and pharmacokinetic study of recombinant human anti-vascular endothelial growth factor in patients with advanced cancer. J Clin Oncol. 2001;19(3):843-850.

23. Scheithauer W, Schmiegel W. Bevacizumab plus oxaliplatin-based regimens for the treatment of colorectal cancer. Onkologie. 2009; 32(7):431-439.

24. Fuchs CS, Marshall J, Mitchell E, et al. Randomized, controlled trial of irinotecan plus infusional, bolus, or oral fluoropyrimidines in first-line treatment of metastatic colorectal cancer: results from the BICC-C Study. J Clin Oncol. 2007;25(30):4779-4786.

25. Fuchs CS, Marshall J, Barrueco J. Randomized, controlled trial of irinotecan plus infusional, bolus, or oral fluoropyrimidines in first-line treatment of metastatic colorectal cancer: updated results from the BICC-C study. J Clin Oncol. 2008;26(4):689-690.

26. Cassidy J, Clarke S, Diaz-Rubio E, et al. XELOX vs FOLFOX-4 as first-line therapy for metastatic colorectal cancer: NO16966 updated results. Br J Cancer. June 28, 2011;105(1):58-64.

27. Giantonio BJ, Catalano PJ, Meropol NJ, et al. Bevacizumab in combination with oxaliplatin, fluorouracil, and leucovorin (FOLFOX4) for previously treated metastatic colorectal cancer: results from the Eastern Cooperative Oncology Group Study E3200. J Clin Oncol. 2007;25(12):1539-1544.

28. Kabbinavar FF, Hambleton J, Mass RD, Hurwitz HI, Bergsland E, Sarkar S. Combined analysis of efficacy: the addition of bevacizumab to fluorouracil/leucovorin improves survival for patients with metastatic colorectal cancer. J Clin Oncol. 2005;23(16):3706-3712.

29. Hurwitz H, Kabbinavar F. Bevacizumab combined with standard fluoropyrimidine-based chemotherapy regimens to treat colorectal cancer. Oncology. 2005;69 Suppl 3:17-24.

30. Chu E. An Update on the Current and Emerging Targeted Agents in Metastatic Colorectal Cancer. Clin Colorectal Cancer. 2012; 11(1):1-13.

31. Kozloff M, Yood MU, Berlin J, et al. Clinical outcomes associated with bevacizumab-containing treatment of metastatic colorectal cancer: the BRiTE observational cohort study. Oncologist. 2009;14(9): 862-870.

32. Samelis GF, Ekmektzoglou KA, Tsiakou A, Konstadoulakis M. The Continuation of Bevacizumab Following Disease Progression in Patients with Metastatic Colorectal Cancer Offers a Survival Benefit. Hepatogastroenterology. 2011;58(112):1968-1971.

33. Shaheen RM, Ahmad SA, Liu W, et al. Inhibited growth of colon cancer carcinomatosis by antibodies to vascular endothelial and epidermal growth factor receptors. Br J Cancer. 2001;85(4):584-589.

34. Ellis LM. Epidermal growth factor receptor in tumor angiogenesis. Hematol Oncol Clin North Am. 2004;18(5):1007-1021.

35. Hecht JR, Mitchell E, Chidiac T, et al. A randomized phase IIIB trial of chemotherapy, bevacizumab, and panitumumab compared with chemotherapy and bevacizumab alone for metastatic colorectal cancer. J Clin Oncol. 2009;27(5):672-680.

36. Tol J, Koopman M, Cats A, et al. Chemotherapy, bevacizumab, and cetuximab in metastatic colorectal cancer. $N$ Engl J Med. 2009; 360(6):563-572.

37. Saltz L, Badarinath S, Dakhil S, et al. Phase III Trial of Cetuximab, Bevacizumab, and 5-Fluorouracil/Leucovorin vs FOLFOX-Bevacizumab in Colorectal Cancer. Clin Colorectal Cancer. 2011. [Epub ahead of print.]

38. De Gramont A, Van Cutsem E, Tabernero J, et al. AVANT: Results from a randomized, three-arm multinational phase III study to investigate bevacizumab with either XELOX or FOLFOX4 versus FOLFOX4 alone as adjuvant treatment for colon cancer. ASCO Meeting Abstracts. February 3, 2011;29(Suppl 4):362.

39. Wolmark N, Yothers G, O'Connell MJ, et al. A phase III trial comparing mFOLFOX6 to mFOLFOX6 plus bevacizumab in stage II or III carcinoma of the colon: Results of NSABP Protocol C-08. ASCO Meeting Abstracts. June 8, 2009;27(15S):A4. 
40. Holash J, Davis S, Papadopoulos N, et al. VEGF-Trap: a VEGF blocker with potent antitumor effects. Proc Natl Acad Sci U S A. 2002;99(17):11393-11398.

41. Stewart MW. Aflibercept (VEGF-TRAP): The Next Anti-VEGF Drug. Inflamm Allergy Drug Targets. 2011;10(6):497-508.

42. Cursiefen C, Chen L, Borges LP, et al. VEGF-A stimulates lymphangiogenesis and hemangiogenesis in inflammatory neovascularization via macrophage recruitment. J Clin Invest. 2004;113(7): 1040-1050.

43. Kim ES, Serur A, Huang J, et al. Potent VEGF blockade causes regression of coopted vessels in a model of neuroblastoma. Proc Natl Acad Sci U S A. 2002;99(17):11399-11404.

44. Teng LS, Jin KT, He KF, Zhang J, Wang HH, Cao J. Clinical applications of VEGF-trap (aflibercept) in cancer treatment. J Chin Med Assoc. 2010;73(9):449-456.

45. Lal D, Park JA, Demock K, et al. Aflibercept exerts antivascular effects and enhances levels of anthracycline chemotherapy in vivo in human acute myeloid leukemia models. Mol Cancer Ther. 2010;9(10):2737-2751.

46. Hu L, Hofmann J, Holash J, Yancopoulos GD, Sood AK, Jaffe RB. Vascular endothelial growth factor trap combined with paclitaxel trikingly inhibits tumor and ascites, prolonging survival in a human ovarian cancer model. Clin Cancer Res. 2005;11(19 Pt 1): 6966-6971.

47. Lockhart AC, Rothenberg ML, Dupont J, et al. Phase I study of intravenous vascular endothelial growth factor trap, aflibercept, in patients with advanced solid tumors. J Clin Oncol. 2010;28(2):207-214.

48. Tew WP, Gordon M, Murren J, et al. Phase 1study of aflibercept administered subcutaneously to patients with advanced solid tumors. Clin Cancer Res. 2010;16(1):358-366.

49. de Groot JF, Lamborn KR, Chang SM, et al. Phase II study of aflibercept in recurrent malignant glioma: a North American Brain Tumor Consortium study. J Clin Oncol. 2011;29(19):2689-2695.

50. Jin K, Shen Y, He K, Xu Z, Li G, Teng L. Aflibercept (VEGF Trap): one more double-edged sword of anti-VEGF therapy for cancer? Clin Transl Oncol. 2010;12(8):526-532.

51. Rudge JS, Holash J, Hylton D, et al. VEGF Trap complex formation measures production rates of VEGF, providing a biomarker for predicting efficacious angiogenic blockade. Proc Natl Acad Sci U S A. 2007;104(47):18363-18370.

52. Reichert JM. Antibody-based therapeutics to watch in 2011. MAbs. 2011;3(1):76-99.

53. Regeneron Pharmaceuticals Inc. Press Release Reporting Two Year Results of Phase 3 Studies with ELYEA ${ }^{\mathrm{TM}}$ (aflibercept) Injection in wet AMD Show Sustained Improvement in Visual Acuity [press release]. Tarrytown, NY: Regeneron; December 5, 2000.
54. Sanofi-Aventis; Regenreron Pharmaceuticals. Study of Intravenous Aflibercept in Combination With FOLFIRI in Japanese Patients With Metastatic Colorectal Cancer. In: ClinicalTrials.gov [Internet]. Bethesda, MD: National Library of Medicine (US). June 15, 2009 [last updated on November 2, 2011]. Available from: http:/clinicaltrials.gov/ ct2/show/NCT00921661. NLM Identifier: NCT00921661.

55. Grothey A, Sugrue MM, Purdie DM, et al. Bevacizumab beyond first progression is associated with prolonged overall survival in metastatic colorectal cancer: results from a large observational cohort study (BRiTE). J Clin Oncol. 2008;26(33):5326-5334.

56. Mass RD, Sarkar S, Holden SN, Hurwitz H. Clinical benefit from bevacizumab $(\mathrm{BV})$ in responding $(\mathrm{R})$ and non-responding $(\mathrm{NR})$ patients (pts) with metastatic colorectal cancer (mCRC). ASCO Meeting Abstracts. May 28, 2005;23(Suppl 16):3514.

57. Kopetz S, Hoff PM, Morris JS, et al. Phase II trial of infusional fluorouracil, irinotecan, and bevacizumab for metastatic colorectal cancer: efficacy and circulating angiogenic biomarkers associated with therapeutic resistance. J Clin Oncol. 2010;28(3):453-459.

58. Groupe Cooperateur Multidisciplinaire en Oncologie (GERCOR). Combination Chemotherapy and Bevacizumab With or Without Erlotinib in Treating Patients With Metastatic Colorectal Cancer That Cannot Be Removed By Surgery. In: ClinicalTrials.gov [Internet]. Bethesda, MD: National Library of Medicine (US). December 14, 2005 [last updated February 6, 2009]. Avaialble from: http://clinicaltrials.gov/ct/ show/NCT00265824. NLM identifier: NCT00265824.

59. Chaplin DJ, Horsman MR, Siemann DW. Current development status of small-molecule vascular disrupting agents. Curr Opin Investig Drugs. 2006;7(6):522-528.

60. Kerbel RS, Kamen BA. The anti-angiogenic basis of metronomic chemotherapy. Nat Rev Cancer. 2004;4(6):423-436.

61. Pohl M, Werner N, Munding J, et al. Biomarkers of anti-angiogenic therapy in metastatic colorectal cancer (mCRC): original data and review of the literature. Z Gastroenterol. 2011;49(10):1398-1406.

62. Loupakis F, Bocci G, Pasqualetti G, et al. Targeting vascular endothelial growth factor pathway in first-line treatment of metastatic colorectal cancer: state-of-the-art and future perspectives in clinical and molecular selection of patients. Curr Cancer Drug Targets. 2010;10(1): 37-45.

63. Matsusaka S, Suenaga M, Mishima Y, et al. Circulating endothelial cells predict for response to bevacizumab-based chemotherapy in metastatic colorectal cancer. Cancer Chemother Pharmacol. 2011;68(3):763-768.
OncoTargets and Therapy

\section{Publish your work in this journal}

OncoTargets and Therapy is an international, peer-reviewed, open access journal focusing on the pathological basis of all cancers, potential targets for therapy and treatment protocols employed to improve the management of cancer patients. The journal also focuses on the impact of management programs and new therapeutic agents and protocols on

\section{Dovepress}

patient perspectives such as quality of life, adherence and satisfaction The manuscript management system is completely online and includes a very quick and fair peer-review system, which is all easy to use. Visit http://www.dovepress.com/testimonials.php to read real quotes from published authors. 